\title{
Spironolactone-Associated Hyponatremic Coma
}

\begin{tabular}{|l|l|l|}
\hline M.M. & Michael M. & Hirschl \\
\hline D. & Dan & Seidler \\
\hline A.N. & Anton N. & Laggner \\
\hline
\end{tabular}

Department of Emergency Medicine, University of Vienna, Austria

Dr. Michael M. Hirschl, Department of Emergency Medicine, New General Hospital, Währinger Gürtel 18-20, A-1090 Vienna (Austria)

Admission

Dear Sir,

Hyponatremia is the most common electrolyte abnormality observed in a general hospitalized population, seen in about $2 \%$ of patients [1]. Common causes of hyponatremia are the syndrome of inappropriate secretion of antidiuretic hormone, water intoxication, advanced renal failure, nephrotic syndrome, congestive heart failure, liver cirrhosis, diarrhea, excessive vomiting and therapy with thiazide diuretica [2]. We present an unusual causes of a hyponatremic coma associated with the use of spironolactone.

Case report: A 93-year-old woman presented to the emergency department with coma of unknown origin. Prior to admission the patient had had severe diarrhea for the last $48 \mathrm{~h}$ with 10-15 watery stools. At 11:00 h the patient was found by a nurse lying on the floor and did not respond to painful stimuli. Her last medication included spironolactone (200

$\mathrm{mg}$ /Aldactone; Boehringer) and oxaze-pam (10 mg/Adumbron; Bender). On presentation the patient was comatose, spontaneously breathing and hemodynamically stable (RR 150/45 mm $\mathrm{Hg}$ ). Physical status established no cardiac murmur, normal vesicular breathing and no peripheral edema. Neurological examination revealed a compromised alertness and a severe reduced response to painful stimuli. Pupillary reaction was delayed but without differences between both sides as well as after painful stimuli all four extremities were moved without differences. According to the neurological status a focal cerebral lesion seemed rather unlikely. Chest X-ray showed no signs of edema. Laboratory data demonstrated a severe hyponatremia with a serum sodium of $104 \mathrm{mmol} / \mathrm{l}$. Urinalysis revealed a sodium of 150 $\mathrm{mmol} / \mathrm{l}$, potassium of $22 \mathrm{mmol} / 1$ and an osmolarity of $360 \mathrm{mosm} / 1$. The urine volume was $1,600 \mathrm{ml} / 24 \mathrm{~h}$. On admission, blood samples were taken to determine thyroid and adrenal hormones. According to the high urine sodium level combined with a severe hyponatremia, a

After $48 \mathrm{~h}$

Discharge $3 / 8 \wedge 85$

Fig. 1. Course of serum sodium ( $\mathrm{mmol} / \mathrm{l}$; $\mathbf{\square})$, urine sodium (mmol/l; $\ddot{\mathrm{U}})$ and aldosterone (pmol/l, M) on admission, $48 \mathrm{~h}$ after admission and on discharge.

hypoaldosteronism was assumed and $0.1 \mathrm{mg}$ fludrocortisone was given. No effect either on renal sodium concentration or urine potassium concentration was observed. According to recent published data [3] we decided to correct hyponatremia slowly to prevent neurological 
complications such as a cerebral pontine myelinolysis. The patient received $150 \mathrm{ml}$ isotonic saline solution per hour. After $24 \mathrm{~h}$ a marked neurological improvement was observed. The patient became alert and orientated and after $72 \mathrm{~h}$ complete restitution of the patient was observed. Serum sodium normalized within $72 \mathrm{~h}$ after admission. Plasma hormone analysis revealed a high serum aldosterone level, whereas vasopressin, cortisol and thyroid hormones were within normal ranges. The course of serum aldosterone, serum and urinary sodium are shown in figure 1 . On admission our patient presented a very confusing picture. The patient suffered from an enteritis-induced hyponatremia. According to the low effective arterial volume and the extrarenal loss of sodium, aldosterone secretion increased. Despite the increased serum aldosterone level and the very low serum sodium, the urinary sodium concentration was extremely high. Furthermore, the application of fludrocortisone did not enhance the renal sodium absorption. Therefore, aldosterone-mediated renal sodium absorption was impaired by spironolactone. After discontinuation of spironolactone, urine sodium concentration decreased despite an enlarged intake of sodium due to continuous infusion of isotonic saline solution. It is without doubt that sprironolactone-associated hyponatremic coma is a rare cause of hyponatremia. However, during episodes of intestinal loss of sodium the spironolactone-induced impairment of aldosterone-mediated resorption of sodium can cause a life-threatening hyponatremia.

References

Man So, Carroll HJ: Disorders of sodium metabolism : Hypernatremia and hyponatremia. Crit Car Med 1992;20:94-103.

Levinsky NG: Fluid and electrolytes: Sodium and water; in Wilson JD, Braunwald E, Isselbacher KJ (eds): Harrison's Principles of Internal Medicine. New York, McGraw-Hill, 1991, pp 278-284.

Sterns RH: The treatment of hyponatremia: First, do no harm. Am J Med 1990;88:557-560. 